\title{
Quality inspection method of layered compacted subgrade and engineering example analysis
}

\author{
Binbin $\mathrm{Xu}^{1,2,3,4^{*}}$ \\ ${ }^{1}$ Tianjin Port Engineering Institute Co. Ltd. of CCCC First Harbor Engineering Co. Ltd., Tianjin, 300222, China \\ ${ }^{2}$ CCCC First Harbor Engineering Co. Ltd., Tianjin, 300461, China \\ ${ }^{3}$ Key Lab. of Geotechnical Engineering of Tianjin, Tianjin, 300222, China \\ ${ }^{4}$ Key Lab. of Geotechnical Engineering, Ministry of Communication, Tianjin, 300222, China
}

\begin{abstract}
The effective detection of layered roller compacted subgrade quality is the key of road engineering quality control. The traditional sand filling compaction method belongs to random sampling point detection method, and it is not easy to detect the subgrade compaction condition below the sand filling pit. Based on the summary of the current domestic and foreign subgrade detection technology, this paper innovatively combines the geological radar method with sand filling method, and through the fixed point detection method. The results show that the traditional sand filling method can directly and quantitatively reflect the compactness of sampling points, while the geological radar can realize the continuous detection, and can judge the compaction layer from the loose state to the interlaminar line after compaction through the geological radar image At the same time, the GPR can identify the under compacted area in the subgrade compaction layer and reflect the overall compaction effect of the subgrade. The detection method of combining the GPR method and sand filling method has obvious technical advantages in the subgrade quality detection.
\end{abstract}

\section{Preface}

In recent years, China's expressway, railway and other infrastructure construction speed has been further improved. In the construction process, affected by the weather, site management, backfill materials, detection means and other factors, it is easy to have excessive rolling layer thickness, unqualified subgrade compaction quality, and even the formation of seepage channel, resulting in subgrade cavity, pavement subsidence and other diseases. Therefore, it is of great significance to timely detect and dynamically adjust the rolling thickness and compactness of Subgrade in the construction process to ensure the engineering quality.

The traditional methods of coring and sampling are inefficient, costly and random. How to take fast, efficient, economic and simple means to detect and scientifically evaluate the compaction quality of subgrade is an urgent technical problem. Relevant technical personnel and scholars have carried out a series of research on subgrade quality detection. Zeng Rong [1] elaborated the test points and process of sand filling method in compactness detection. The method proposed by Feng Li Ying can reflect the actual compaction degree of soil rock mixture. Li Yalong [3] applied the portable falling weight deflectometer (PFWD) in the compaction detection of Subgrade of Changlin expressway, and achieved good results. Peng Rui [4] discussed the application characteristics and rules of Rayleigh wave method in detecting subgrade compactness based on engineering examples. However, the existing detection methods still do not meet the requirements of accuracy, convenience and comprehensiveness. Therefore, based on the investigation and summary of various detection technologies, this paper analyzes the application scope, advantages and disadvantages of each detection technology, puts forward a comprehensive detection technology combining sand filling method and electromagnetic wave method, and carries out field detection research in a subgrade project, which has achieved good results.

\section{Common detection methods of subgrade rolling effect}

As the most commonly used control index of subgrade compaction effect, the evaluation index can be obtained through a variety of technical methods, which is mainly divided into two categories: damage detection and nondestructive testing. The following summarizes and analyzes the commonly used damage detection and nondestructive testing technology of subgrade.

\subsection{Damage detection method}

At present, the commonly used damage detection methods at home and abroad are cutting-ring method,

\footnotetext{
* Corresponding author: xubinbin@tpei.com.cn
} 
sand filling method, nuclear densitometer method and so on.

The cutting-ring method [5] is a method to obtain the compactness of measuring points by taking soil with ring knife, drying and weighing. It has the advantage of simple apparatus, and is suitable for soft soil layer. It is not suitable for soil containing gravel, crushed stone and poorly graded soil. Moreover, the test results are relatively dependent on the professional quality of the testing personnel, and are easily affected by subjective factors and cause great errors Poor.

Nuclear densitometer method [5] is a method to measure the material according to the number of rays and compactness returned by Compton scattering. By using this method, the dry and wet density, moisture content, porosity, compactness and other indicators of the material can be obtained. However, due to the radioactive substances contained in the equipment, higher safety requirements are put forward for the transportation and on-site personnel handling process, and it is required to be used in advance The operation steps are relatively complex.

Compared with the ring knife method and the nuclear densitometer method, the sand filling method is a compaction detection method with controllable operation process and accurate detection results. It is often used to replace and measure the volume of the hole by using the sand with uniform particle size. The compaction of the soil at the measuring point can be obtained by calculation, but it also has some shortcomings, such as low operation efficiency and large random sampling.

\subsection{NDT Method}

At present, the common nondestructive testing methods include electrical method, wave velocity method, ground penetrating radar method and so on.

Electrical measurement method [6] is mainly used to determine the geological structure by studying the distribution law of electric field, and to understand the stratum changes in the target area by determining the electric property difference of stratum. It is mostly used in the disease detection of subgrade. Engineering application examples show that this method has some shortcomings, such as low accuracy, slow detection efficiency, etc., and it is difficult to establish the connection with the stratum mechanics index. These phenomena have great influence on the quality of subgrade The field application effect of the electric measurement method is discussed.

The wave velocity method [7] can determine the physical and mechanical indexes of the stratum, and can be divided into surface wave method and cross hole wave velocity method according to the application scope. The surface wave method [8] uses the propagation characteristics of Rayleigh surface wave along the ground surface to excite and receive signals on the subgrade surface, According to the surface wave propagation law, the wave velocity of each layer is inversed; the cross hole wave velocity method [9] uses two holes or two opposite surfaces to receive shear waves, and calculates the propagation velocity of shear waves of two sensors on two holes or two surfaces. Wave velocity method is used to distinguish strata and determine their physical and mechanical indexes. It has fast testing speed, but low resolution and is easily affected by frequency, water content and testing conditions.

Electromagnetic wave method [10] is to transmit electromagnetic wave to Subgrade through radar antenna, and determine the junction position of each structural layer by recording the position of reflected wave. Compared with the above two methods, the application of GPR method is more convenient, and the whole operation process is non-destructive, which can meet the research requirements of most engineering projects. However, GPR method can only determine the density of subgrade compaction, and it is difficult to obtain specific compaction effect index.

\section{Comprehensive detection technology of sand filling method and electromagnetic wave method}

Through the investigation and field test, it is found that although the nuclear density meter method, ring knife method, sand filling method and other destructive testing methods can be used to measure the compaction index of subgrade more accurately, the testing process is often random sampling test, and the results are random, so it is difficult to make a comprehensive evaluation of the compaction effect of Subgrade in the field; while the wave velocity method, geological radar method and other nondestructive testing methods are used Although the method can quickly and comprehensively measure the change of stratum properties, it is difficult to obtain quantitative compaction index. Therefore, this paper considers the combination of destructive testing and nondestructive testing, using a comprehensive testing method based on sand filling method and electromagnetic wave method, in order to achieve the purpose of rapid, accurate and comprehensive testing.

\subsection{Principle of sand filling method}

The sand filling method is a method to measure the volume of the test hole by using the clean and uniform sand with the particle size of $0.30-0.60 \mathrm{~mm}$, according to the principle that the unit volume weight of the sand freely falls into the test hole from a certain height remains unchanged, and calculate the measured dry density of the sample by combining with the water content of the aggregate. The specific calculation method is as follows:

$$
\begin{aligned}
& \rho_{0}=\mathrm{MP} \times \rho_{\mathrm{s}} \div \mathrm{MS} \\
& \rho_{0}=\mathrm{MP} \times \rho_{\mathrm{s}} \div \mathrm{MS} \\
& \rho_{\mathrm{d}}=\mathrm{MP} \div(1+\omega \%)
\end{aligned}
$$


Where: $\rho 0$ is sample density; MP is sample mass; MS is full injection mass; $\rho \mathrm{s}$ is full injection standard density; $\rho \mathrm{D}$ is sample dry density; $\omega$ is water content.

\subsection{Detection principle of electromagnetic wave method}

Electromagnetic wave method is based on the propagation difference of electromagnetic pulse in different underground media, which is mainly manifested as interface reflection. With the help of radar antenna, it transmits and receives the reflection echo of different media interface. According to the received echo time, amplitude, waveform and frequency information, it determines the structure of underground media and the buried depth of interface. Because this method is fast, efficient, accurate, low cost, no damage and other advantages, it can be used in the whole process of highway construction quality monitoring.

The ground penetrating radar can qualitatively detect the change of compactness of subgrade before and after compaction. The difference of radar wave frequency between undisturbed soil profile and vibro-flotation profile reflects the difference of compactness.

\subsection{Comprehensive control technology of subgrade quality}

In order to effectively control the subgrade quality in the construction process, this paper puts forward a comprehensive detection technology of subgrade layered rolling based on sand filling method and electromagnetic wave method, that is, when the subgrade soil layer is loose and rolled, the sand filling method is used to measure the subgrade compactness, and the subgrade compactness is quantitatively analyzed. At the same time, the continuous detection of subgrade compactness with the help of geological radar can be qualitatively evaluated.

\section{Engineering application examples and analysis}

\subsection{Sand filling method}

Before the subgrade construction of a mountainous expressway, indoor tests are carried out on the soil samples to be used to determine the maximum dry density and optimal moisture content of the soil samples. In addition, on-site rolling test section is set to obtain the maximum loose paving thickness of the soil samples to meet the design compactness requirements and the compaction times under the rolling counterweight. The final process parameters for the subgrade of this section are: rolling The counterweight is $26 \mathrm{t}$, and the thickness of loose paving is $25 \mathrm{~cm}$. Firstly, the compaction layer is about $20 \mathrm{~cm}$ thick, and then the compaction layer is about $20 \mathrm{~cm}$ thick.

In the process of subgrade rolling construction, every layer of subgrade is rolled, sand filling method is used to detect the compactness of subgrade according to the requirements of specification [11]. There are two compactness detection points every $200 \mathrm{~m}$ for a single double lane, as shown in Figure 1. The compactness of one of the rolled subgrade is tested, and the test results are shown in Table 1. It can be seen from table 1 that it meets the design requirements of compactness greater than $93 \%$. According to the compactness test results of sand filling method, the subgrade quality is qualified, and the next layer construction can be carried out.
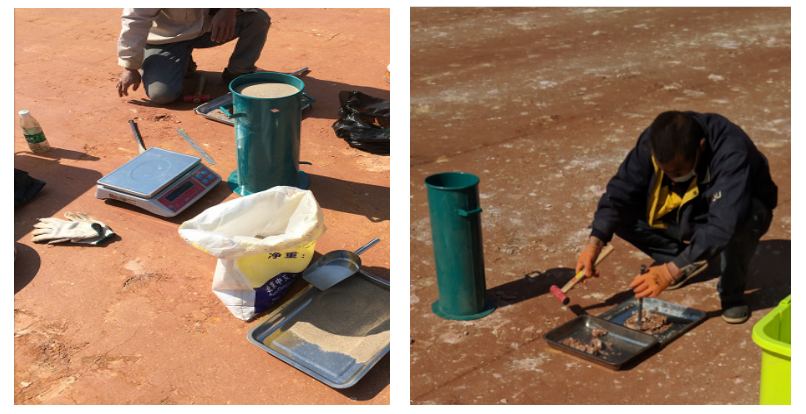

Fig.1 Subgrade compaction detection on site

Table 1 Results of compaction test

\begin{tabular}{ccccc}
\hline NO. & Mass $/ \mathrm{kg}$ & \multicolumn{2}{c}{ WC/\% Density $/ \mathrm{g} \bullet \mathrm{cm}^{-3}$} & $\begin{array}{c}\text { compaction } \\
/ \%\end{array}$ \\
\hline 1 & 8.791 & 7.2 & 1.99 & 93.4 \\
2 & 9.378 & 7.2 & 2.02 & 94.8 \\
\hline
\end{tabular}

\subsection{Ground penetrating radar method}

Laurel SIR-20 ground penetrating radar is used for Subgrade rolling quality detection, which is equipped with $900 \mathrm{MHz}$ high configuration shielded antenna and $400 \mathrm{MHz}$ intermediate frequency shielded antenna, as shown in Figure 2. The detection depth of $900 \mathrm{MHz}$ is about $80 \mathrm{~cm}$, and that of $400 \mathrm{MHz}$ is about $150 \mathrm{~cm}$.
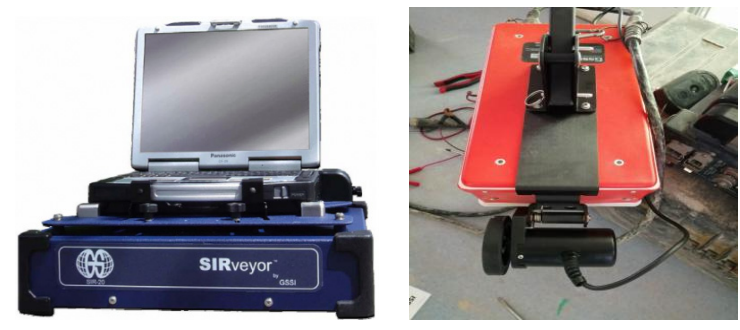

Fig.2 Ground penetrating radar image

(1) Detection of loose layer

The ground penetrating radar (GPR) is used to scan and test the loose layer before rolling. During the test, two parallel measuring lines with a length of $10 \mathrm{~m}$ and a spacing of $20 \mathrm{~m}$ are arranged. The field test situation is shown in Figure 3.

In the field test, $400 \mathrm{MHz}$ antenna parameters are set as follows: transmitting power $100 \mathrm{kHz}$, time window $20 \mathrm{~ns}$, single channel wave sampling points 512 , time trigger mode, scanning channels per second 100 , gain points 3 , gain values $12,20,25$, low-pass filter, $800 \mathrm{MHz}$, high pass filter $200 \mathrm{MHz}$, static superposition 3, relative permittivity $8 ; 900 \mathrm{MHz}$ antenna test parameters are set 
as transmitting power $100 \mathrm{kHz}$, time window $15 \mathrm{~ns}$ Single channel wave sampling points 512 , time triggered mode, 100 channels per second, gain points 1 , gain value 12 , low-pass filter, $2000 \mathrm{MHz}$, high pass filter $450 \mathrm{MHz}$, static superposition 5 , relative permittivity 8 . The radar detection image of the loose layer is shown in Fig. 4 and Fig. 5.

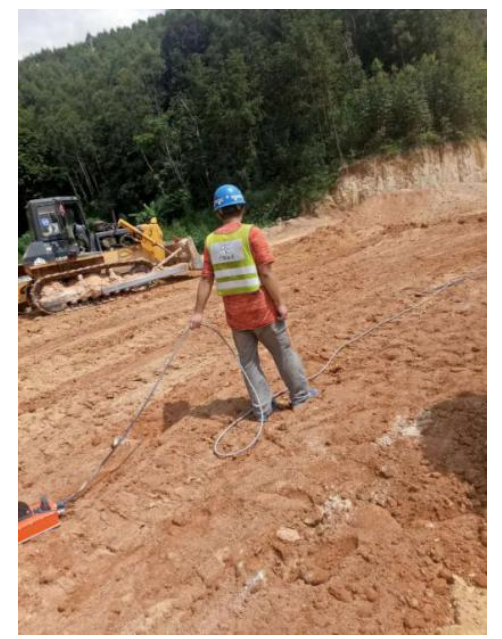

Fig.3 On-site inspection photos

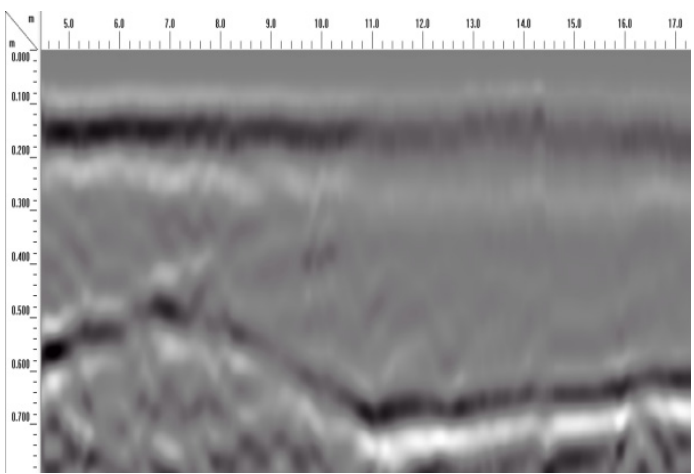

(a ) $400 \mathrm{MHz}$

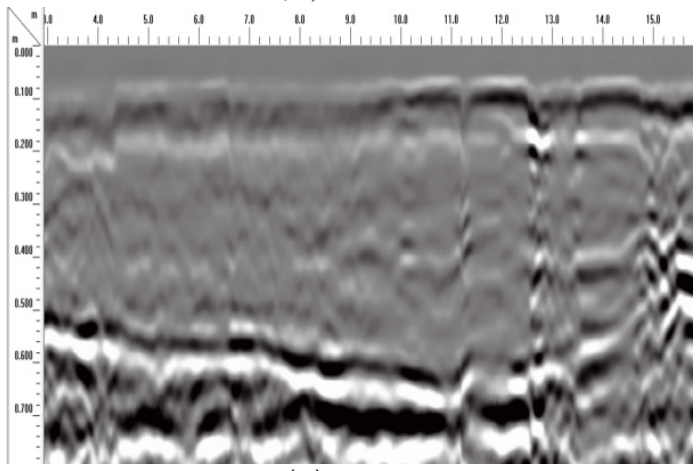

(b) $900 \mathrm{MHz}$

Fig.4 Radar image of loose layer of line one

Through the analysis and comparison of the GPR images in Fig. 4 and Fig. 5, we can see that there is electromagnetic wave strong reflection signal in the lower part of the image, and the same phase axis is continuous, showing obvious interface reflection characteristics. Therefore, the GPR can clearly determine the interface between the loose layer and the lower compacted layer, indicating that there are obvious physical properties in the upper and lower interface soil layers due to the different soil density and particle spatial arrangement It also provides a good theoretical basis for the application of GPR Detection Technology in subgrade compaction quality detection. Comparing the two frequency antenna images of line 1 and line 2, it can be found that there are many groups of disordered signals in the $900 \mathrm{MHz}$ antenna image on the upper part of the interface, while the reflection signal in the 400 $\mathrm{MHz}$ antenna image is weak. The lower part of the interface is uniform waveform, and there is no strong reflection signal. It is the upper part of the lower compacted subgrade, which is qualified by the compaction sampling inspection of sand filling method, which is consistent with the site conditions.

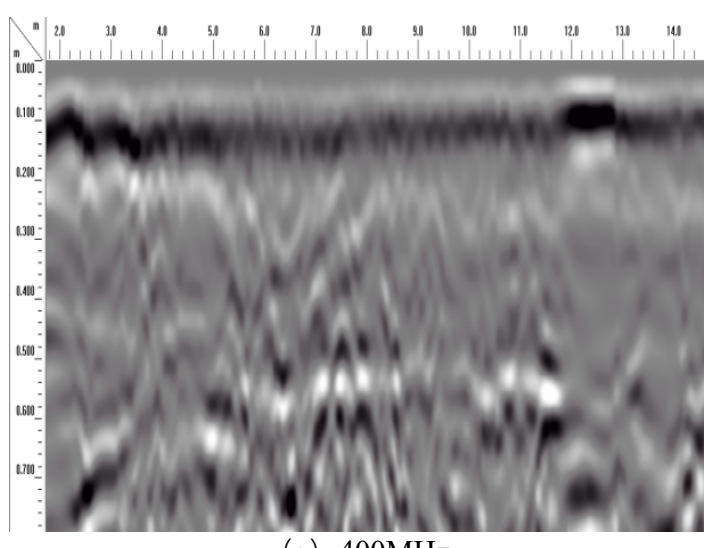

(a) $400 \mathrm{MHz}$

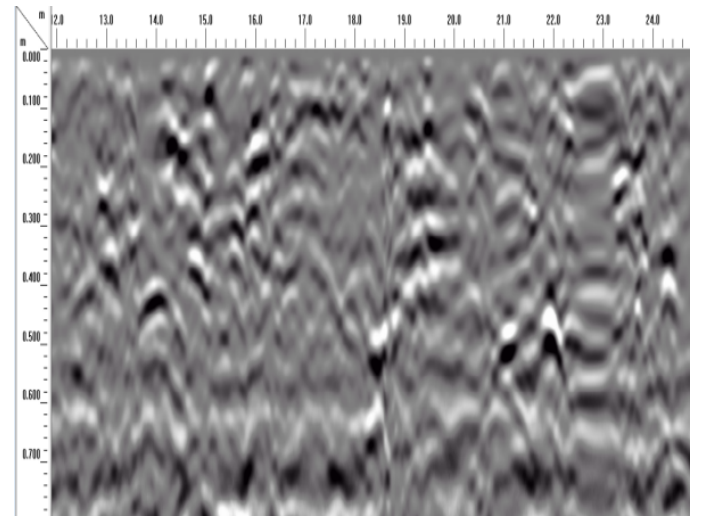

(b) $900 \mathrm{MHz}$

Fig.5 Radar image of loose layer of line two

The resolution of $900 \mathrm{MHz}$ antenna is higher than that of $400 \mathrm{MHz}$ antenna. It can be seen from Fig. 4 and Fig. 5 that both kinds of antennas can meet the requirements of subgrade compaction quality detection; the depth of $400 \mathrm{MHz}$ antenna is larger than that of $900 \mathrm{MHz}$ antenna. If it is necessary to detect multi-layer compacted subgrade, $400 \mathrm{MHz}$ antenna can be used, while $900 \mathrm{MHz}$ antenna is recommended to detect single-layer subgrade to obtain more compaction information.

(2) Detection of compacted layer

The ground penetrating radar (GPR) is used to detect the compacted subgrade passing the sand filling method. Considering the shallow detection depth, the $900 \mathrm{MHz}$ antenna is used. The time window of parameter setting is $15 \mathrm{~ns}$, the number of single channel wave sampling points is 512 , the number of channels per second is 100 , the number of gain points is 1 , the gain value is 12 , the 
low-pass filter is $2000 \mathrm{MHz}$, and the high pass filter is $450 \mathrm{MHz}$. Two lines, line a and line B, are arranged for on-site inspection. The measuring line is parallel to the longitudinal direction of subgrade, with a spacing of about $15 \mathrm{~m}$ and a length of $50 \mathrm{~m}$. The images of line a and line B are shown in Fig. 6 and Fig. 7.

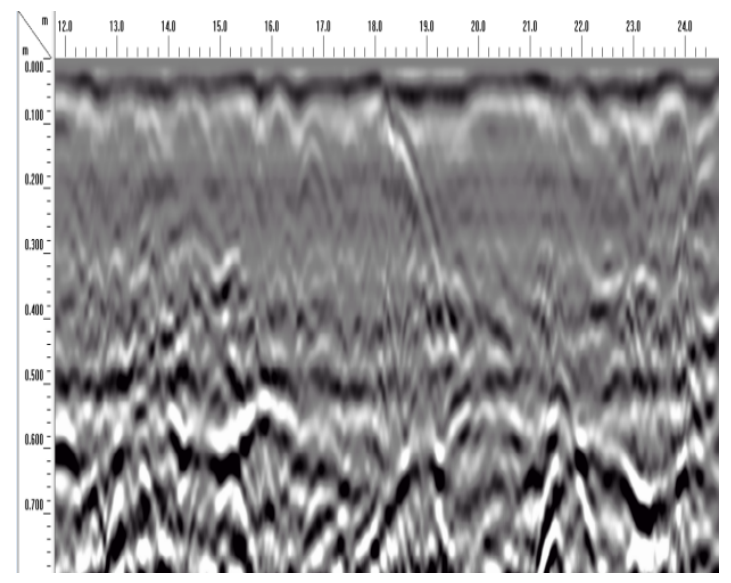

Fig.6 Image of compacted layer of line A

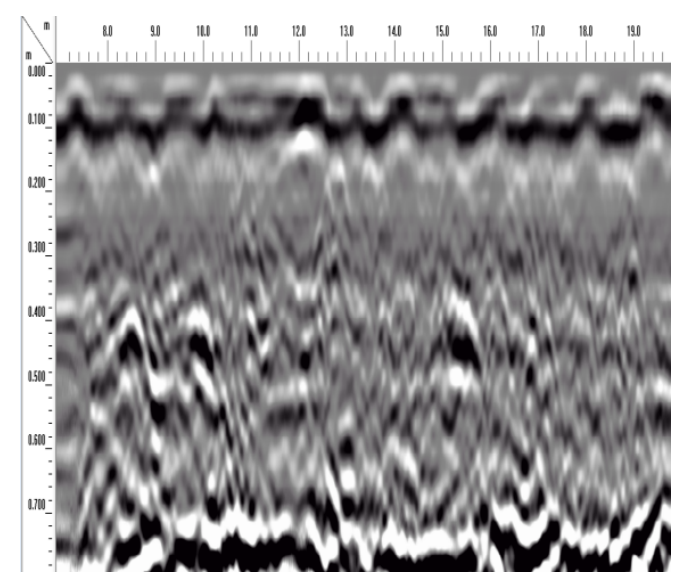

Fig.7 Image of compacted layer of line B

According to the GPR images of the compacted layer in Fig. 6 and Fig. 7, the reflection energy of the radar waveform is relatively uniform when the compacted layer is $35 \mathrm{~cm}$ in depth, and there is no interface feature, which indicates that the soil layer is dense and close to the compaction degree at the detection point, and meets the quality requirements; when the depth is below $35 \mathrm{~cm}$, the two measuring lines show strong reflection signals, and there is a continuous phase axis at the depth of $60 \mathrm{~cm}$ at the measuring line $\mathrm{B}$, and the image reflects that there is local reflection in the lower soil layer In the loose area, the compaction quality of the compacted soil layer is poor, and the interface of the soil layer is still obvious, which belongs to undercompaction.

The reasons for under compaction in Fig. 6 and Fig. 7 may be as follows: 1) the process parameters of the test section are not strictly implemented in the construction process; 2) there is a great difference between the filling soil and the soil of the test section; 3 ) in the subgrade construction, in order to pursue efficiency, save time and cost, the construction personnel often increase the thickness of the loose layer, because the compaction work diffuses with the depth energy in the soil layer, resulting in more than a certain depth However, the upper soil layer is compacted to form a dense shell, and part of the deep soil layer which is not fully compacted will continue to consolidate under the action of self weight, even partial void.

To sum up, even if the sand filling tube with the largest specification (the maximum detection depth is $40 \mathrm{~cm}$ ) is used in the compaction detection of sand filling method, it is still difficult to conduct a comprehensive quality detection on the super thick rolled layer; as the foundation of the road, the subgrade is a concealed project, and the process quality control is particularly important. This paper adopts the technical means of combining the compaction detection of sand filling method with the subgrade detection of geological radar method It can realize continuous and deep detection of subgrade compaction and achieve the purpose of comprehensive and strict control of subgrade quality.

\section{Conclusions}

(1) On the basis of summing up the application scope, advantages and disadvantages of subgrade detection technology, this paper uses sand filling compaction test and geological radar method to comprehensively apply to Subgrade detection. The former is quantitative analysis, and the latter is qualitative evaluation, which can realize continuous and deep detection of subgrade compaction quality, and the effect is remarkable;

(2) Both $400 \mathrm{MHz}$ if antenna and $900 \mathrm{MHz} \mathrm{HF}$ antenna of GPR can be used for subgrade quality detection. The detection depth of $400 \mathrm{MHz}$ antenna is larger than that of $900 \mathrm{MHz}$ antenna, and the detection accuracy of $900 \mathrm{MHz}$ antenna is higher than that of $400 \mathrm{MHz}$ antenna. The antenna frequency can be determined according to the actual demand;

(3) The radar image shows that the boundary of the loose layer is obvious before compaction, and disappears after compaction, and the physical parameters of the upper and lower layers tend to be the same;

(4) For the rolled soil layer, according to whether the radar image shows strong reflection signal, the overall compaction effect of soil layer can be qualitatively judged.

\section{References}

1. Z. Rong, Traffic world, 2019 (29) 78-79

2. L.Y. Feng, Traffic world, 2020 (26) 69-70

3. Y. Li,Shanxi Commu. Technology, 2020 (04) 73-75

4. P. Rui, Northern Comm., 2018 (05) 102-105

5. D. Su, Fujian building materials, 2020 (08) 17-19

6. L. Rui, C. Ai, F. Kong, Acta engineering geology, 2020,28 (01) 51-59

7. F.F. Huang, Traffic world, 2016 (25) 40-41

8. Y.S. Cheng, Z.L. Zhu, Q.L. Zhang, L.J. Wang, Railway construction, 2007 (01) 74-76

9. Q.H. Sun, Chongqing Jiaotong University, 2010 
10. Site test code for Subgrade and pavement of highway, JTG3450-2019 